\title{
Cosmological Duality in Four Time and Four Space Dimensions
}

\author{
M. Medina, J. A. Nieto, P. A. Nieto-Marín \\ Facultad de Ciencias Físico-Matemáticas de la Universidad Autónoma de Sinaloa, Culiacán, Sinaloa, México \\ Email:m.m.walldez@gmail.com,niet@uas.edu.mx, janieto1@asu.edu,pedronieto.fcfm@uas.edu.mx
}

How to cite this paper: Medina, M., Nieto, J.A. and Nieto-Marín, P.A. (2021) Cosmological Duality in Four Time and Four Space Dimensions. Journal of Modern Physics, 12, 1027-1039. https://doi.org/10.4236/jmp.2021.127064

Received: March 22, 2021

Accepted: May 24, 2021

Published: May 27, 2021

Copyright (c) 2021 by author(s) and Scientific Research Publishing Inc. This work is licensed under the Creative Commons Attribution International License (CC BY 4.0).

http://creativecommons.org/licenses/by/4.0/

\begin{abstract}
We describe a duality transformation in a cosmological model of four time and four space dimensions $((4+4)$-dimensions). In particular, we show that via the Fourier transform, at the level of the zero-point energy of quantum mechanics and the de Sitter space, a Gaussian distribution in four dimensions leads to a dual Gaussian distribution also in four dimensions, with duality transformation $\sigma \rightarrow \frac{1}{\sigma}$, in the standard deviation $\sigma$. Moreover, we show that as a consequence of such a duality in $\sigma$ a duality of the cosmological constant $\Lambda$ can be obtained. Finally, we comment on the possibility that both the oriented matroid theory as well as the surreal number theory are related to the formalism presented in this work.
\end{abstract}

\section{Keywords}

Cosmology, (4 + 4)-Dimensional Gravitational Theory, Classical and Quantum Gravity

\section{Introduction}

It is known that the $(5+5)$-dimensional space-time (five time and five space dimensions) is a common signature to both type IIA strings and type IIB strings [1]. In fact, versions of $M$-theory [2] lead to type IIA and to type IIB string in space-time of signatures $(5+5)$. It turns out that by duality transformations string theories of signatures $(5+5)$ can be related to other string signatures such as $(1+9)[3]$.

Just as the $(1+3)$-dimensional signature can be considered as a reduced world of the de Sitter $(1+4)$-dimensional or anti-de Sitter $(2+3)$-dimensional signatures via the cosmological constant $\Lambda>0$ and $\Lambda<0$ (see Refs [4] [5] and 
references therein), respectively, here, we shall assume that up to two cosmological constants, the $(4+4)$-world emerges from $(5+5)$-dimensional world. Specifically, we show that via a Fourier transform a complete duality symmetry of the standard deviation

$$
\sigma^{2} \leftrightarrow \frac{1}{\sigma^{2}}
$$

of a Gaussian of distribution of 4-space coordinates associated with the de Sitter space (anti-de Sitter) and the vacuum zero-point energy yields to a Gaussian of 4 -time coordinates of the same vacuum scenario. This is in fact one of our main contribution and its importance emerges when we notice that only in 4-dimensions such a totally duality symmetry is achieved. In the process, we discover that in 4-dimensions the cosmological constant $\Lambda>0$ associated with the de Sitter space $(\Lambda<0$, anti de Sitter space) is dual to the cosmological constant $\Lambda_{P}=\frac{3}{l_{P}}$, where $l_{P}=\left(\frac{\hbar G}{c^{3}}\right)^{1 / 2}$ is the Planck length, with $G$ the gravitational Newton constant, $\hbar$ the Planck constant and $c$ the light velocity.

There are at least two frameworks where a $(4+4)$-world has emerged as interesting physical scenario. First, it has been proved [6] that the Dirac equation in $(4+4)$-dimensions admit a Majorana-Weyl physical spinor state with only 8 real components which can be identified with the 4-complex components of the usual electron components. Second, in Ref. [7] it has been shown that a general Kruskal-Szekeres transform, in black-hole physics, implies 8 hidden regions instead of just 4-regions as it is usually believed. It turns out that this 8-regions admit better interpretation in a world of $(4+4)$-dimensions.

Technically, this work is organized as follows. In Section 2, we consider the geodesic of a point particle in the de Sitter (anti-de Sitter) space and show that the classical harmonic oscillator equation in 4-dimensions emerges. In Section 3 , we quantize the system obtained in the previous section and focus in the case of zero-point energy showing that this case admits a Gaussian distribution solution. While in Section 4, by the use of the Fourier transform we investigate a duality scenario. Thus combining the zero-point energy and de Sitter vacuum space we discover a duality of the cosmological constant. Finally in Section 5, we express a number of final remarks. In particular, from our analysis of the Gaussian distribution from 4-space dimensions to 4-time dimensions we conclude that the space-time involved may be considered corresponds to a (4 + 4)-dimensional spacetime. Moreover, we briefly comment about the possibility that, for further work, the mathematical structures of matroid theory and surreal number theory may be interesting routes for a connection with our approach.

\section{Geodesic Equation of the de Sitter (Anti-de Sitter) Space}

Let us start recalling some geometrical aspects of the de Sitter space (or anti de Sitter space). In particular, it is well known that the Christoffel symbols in the de 
Sitter space satisfy the equation

$$
\Gamma_{\alpha \beta}^{\mu}=\frac{x^{\mu}}{l_{0}^{2}} g_{\alpha \beta} .
$$

Here, the metric $g_{\alpha \beta}$ is given by

$$
g_{\alpha \beta}=\delta_{\alpha \beta}+\frac{x_{\alpha} x_{\beta}}{l_{0}^{2}-x^{\tau} x^{\sigma} \delta_{\tau \sigma}},
$$

and $l_{0}^{2}$ is determined by

$$
l_{0}^{2}=x^{\tau} x^{\sigma} \delta_{\tau \sigma}+\left(x^{5}\right)^{2},
$$

where $\delta_{\tau \sigma}$ is the Kronecker delta (see Ref. [8] and references therein). The indices $\alpha, \beta, \cdots$ etc run from 1 to 4 . Thus, using (1) the geodesic equation

$$
\ddot{x}^{\mu}+\Gamma_{\alpha \beta}^{\mu} \dot{x}^{\alpha} \dot{x}^{\beta}=0 \text {, }
$$

yields

$$
\ddot{x}^{\mu}+\frac{x^{\mu}}{l_{0}^{2}} g_{\alpha \beta} \dot{X}^{\alpha} \dot{x}^{\beta}=0
$$

In general, for arbitrary variable $A(\tau)$ one defines $\dot{A} \equiv \frac{\mathrm{d} A}{\mathrm{~d} \tau}$ and $\ddot{A} \equiv \frac{\mathrm{d}^{2} A}{\mathrm{~d} \tau^{2}}$. Further, the line element is given by $c^{2} \mathrm{~d} \tau^{2}=g_{\alpha \beta} \mathrm{d} x^{\alpha} \mathrm{d} x^{\beta}$. Thus, one sees that $g_{\alpha \beta} \dot{X}^{\alpha} \dot{X}^{\beta}=c^{2}$ and therefore (5) becomes

$$
\ddot{x}^{\mu}+\frac{c^{2} x^{\mu}}{l_{0}^{2}}=0 .
$$

One recognizes in (6) the classical harmonic oscillator equation in 4-dimensions, with $\kappa=\frac{c^{2}}{l_{0}^{2}}$ as the spring constant.

\section{Quantization; Zero-Point "Energy"}

Let us now quantize the system described by the expression (6) from which one finds the constant of motion

$$
\varepsilon=\frac{\mathcal{P}^{\tau} \mathcal{P}^{\sigma} \delta_{\tau \sigma}}{2}+\frac{\boldsymbol{x}^{\tau} \boldsymbol{X}^{\sigma} \delta_{\tau \sigma}}{2 l_{0}^{2}},
$$

with $\mathcal{P}^{\tau}=\frac{\dot{X}^{\tau}}{c}$. Therefore, at the quantum level one promotes the quantities $\varepsilon$ and $\mathcal{P}^{\tau}$ by the operators $\hat{\varepsilon}=-i l_{P} \frac{\partial}{c \partial \tau}$ and $\hat{\mathcal{P}}_{\mu}=-i l_{P} \frac{\partial}{\partial x^{\mu}}$, respectively. Here, we assume that $l_{P}$ is the Planck length, namely $l_{P}=\left(\frac{\hbar G}{c^{3}}\right)^{1 / 2}$. Note that the reason to write the quantum operators $\hat{\varepsilon}$ and $\hat{\mathcal{P}}_{\mu}$ in terms of $l_{p}$ rather than $\hbar$ can be traced back to the geodesic Equation (4) which is independent of the test particle mass and therefore the quantities $\varepsilon$ and $\mathcal{P}^{\sigma}$ that play the analo- 
gue role of energy and momentum, are dimensionless. Thus, the formula (7) leads to the Schrödinger equation

$$
\hat{\varepsilon} \psi=\frac{\hat{\mathcal{P}}_{\tau} \hat{\mathcal{F}}_{\sigma} \delta^{\tau \sigma}}{2} \psi+\frac{x^{\tau} X^{\sigma} \delta_{\tau \sigma}}{2 l_{0}^{2}} \psi
$$

One may assume the usual proposal for the physical state $\psi=\mathrm{e}^{i \frac{c \varepsilon \tau}{l_{P}}} \varphi\left(x^{\mu}\right)$. This leads to

$$
\varepsilon \varphi=\frac{\hat{\mathcal{P}}_{\tau} \hat{\mathcal{P}}_{\sigma} \delta^{\tau \sigma}}{2} \varphi+\frac{x^{\tau} X^{\sigma} \delta_{\tau \sigma}}{2 l_{0}^{2}} \varphi
$$

Of course, in analogy to the usual harmonic oscillator equation, (9) must imply that $\varepsilon$ is quantized. Since one is mainly interested in the lowest energy state, here we shall be concerned only with the zero-point "energy". For this purpose for each coordinate in $x^{\mu}=(x, y, z, \xi)$ one must have the formula

$$
\varepsilon_{(x, y, z, \xi)}=\frac{l_{P} \omega}{2},
$$

with $\omega=\frac{1}{l_{0}}$ and

$$
\varphi=\varphi_{x}(x) \varphi_{y}(y) \varphi_{z}(z) \varphi_{\xi}(\xi) .
$$

Thus, in the zero-point "energy", for each coordinate in $(x, y, z, \xi)$ one must have the equation

$$
\frac{l_{P} \omega}{2}=-\frac{l_{P}^{2}}{2} \frac{1}{\varphi_{x}} \frac{\mathrm{d}^{2} \varphi_{x}}{\mathrm{~d} x^{2}}+\frac{\omega^{2} x^{2}}{2},
$$

where $\varphi_{x}$ denotes the function $\varphi$ evaluated for each coordinate in $(x, y, z, \xi)$. Solving (12) for $\varphi_{x}$ one discovers that

$$
\varphi_{x} \sim \mathrm{e}^{-\frac{\sigma^{2} x^{2}}{2}},
$$

where

$$
\sigma=\left(\frac{\omega}{l_{P}}\right)^{1 / 2}=\left(\frac{1}{l_{P} l_{0}}\right)^{1 / 2}
$$

is the inverse of the standard deviation. Following similar steps, for the other coordinates $y, z, \xi$ one finally discovers that

$$
\varphi=\frac{\sigma^{q}}{(2 \pi)^{q / 2}} \mathrm{e}^{-\frac{\sigma^{2}\left(x^{2}+y^{2}+z^{2}+\xi^{2}\right)}{2}}
$$

or

$$
\varphi=\frac{\sigma^{q}}{(2 \pi)^{q / 2}} \mathrm{e}^{-\frac{\sigma^{2}\left(x^{\mu} x^{v} \delta_{\mu v}\right)}{2}} .
$$

Here, $q$ is a positive integer number to be determined below. Of course, (15) (or (16)) corresponds to Gaussian distribution in 4-dimensions. 


\section{Duality and the Fourier Transform}

It turns out that the Fourier transform of (15) or (16) is given by

$$
* \varphi=\frac{(2 \pi)^{q / 2}}{\sigma^{q}} \mathrm{e}^{-\frac{t^{\tau} t^{\sigma} \delta_{\tau \sigma}}{2 \sigma^{2}}} .
$$

In fact, let us show in some detail that (15) (or (16)) implies (17). For this purpose let ${ }^{*} \mathcal{F}(t)$ be the Fourier transform that sends us from the one dimensional $x$-space to the $t$-space

$$
{ }^{*} \mathcal{F}(t)=\int_{-\infty}^{\infty} \mathrm{e}^{i t x} \mathcal{F}(x) \mathrm{d} x
$$

Here the function $\mathcal{F}(x)$ is given by $\mathcal{F}(x)=\frac{\sigma}{\sqrt{2 \pi}} \mathrm{e}^{-\frac{\sigma^{2} x^{2}}{2}}$, where typically the factor $\frac{\sigma}{\sqrt{2 \pi}}$ is introduced for normalizing the function $\mathcal{F}(x)$. The Fourier transform ${ }^{*} \mathcal{F}(t)$ is obtained as follows; by definition one has

$$
{ }^{*} \mathcal{F}(t)=\frac{\sigma}{\sqrt{2 \pi}} \int_{-\infty}^{\infty} \mathrm{e}^{i t x} \mathrm{e}^{-\frac{\sigma^{2} x^{2}}{2}} \mathrm{~d} x,
$$

then, completing the squares one finds

$$
{ }^{*} \mathcal{F}(t)=\frac{\sigma}{\sqrt{2 \pi}} \int_{-\infty}^{\infty} \mathrm{e}^{-\frac{1}{2}\left(\sigma x-\frac{i t}{\sigma}\right)^{2}} \mathrm{e}^{-\frac{t^{2}}{2 \sigma^{2}}} \mathrm{~d} x
$$

Now, introducing the variable $u=\sigma x-\frac{i t}{\sigma}$ so that the differential remains as $\mathrm{d} u=\sigma \mathrm{d} x$ one finds that the Fourier transform (20) becomes

$$
{ }^{*} \mathcal{F}(t)=\frac{1}{\sqrt{2 \pi}} \mathrm{e}^{-\frac{t^{2}}{2 \sigma^{2}}} \int_{-\infty}^{\infty} \mathrm{e}^{-\frac{u^{2}}{2}} \mathrm{~d} u
$$

Making the integral, one ends up with

$$
{ }^{*} \mathcal{F}(t)=\mathrm{e}^{-\frac{t^{2}}{2 \sigma^{2}}}
$$

Thus, the Fourier transform of a Gaussian distribution in the $x$-space, with standard deviation $\frac{1}{\sigma}$ is another Gaussian distribution, but now in the $t$-space and with standard deviation $\sigma$. This is of course well known fact which is expressed in simply words as; the Fourier transform of Gaussian is another Gaussian. However, in the general case of higher dimensions we shall put spacial attention to the duality relation $\sigma \leftrightarrow \frac{1}{\sigma}$ of the deviation standard.

So, let us generalize the above procedure to any dimension $d$. One has

$$
\begin{aligned}
& { }^{*} \mathcal{F}\left(t_{1}, \cdots, t_{d}\right)=\frac{\sigma^{q}}{(2 \pi)^{q / 2}} \int_{-\infty}^{\infty} \cdots \int_{-\infty}^{\infty} \mathrm{e}^{-\frac{1}{2}\left(\sigma x^{1}-\frac{i t^{1}}{\sigma}\right)^{2}} \cdots \mathrm{e}^{-\frac{1}{2}\left(\sigma x^{d}-\frac{i t^{d}}{\sigma}\right)^{2}} \\
& \times \mathrm{e}^{-\frac{\left(t^{1}\right)^{2}}{2 \sigma^{2}} \cdots-\frac{\left(t^{d}\right)^{2}}{2 \sigma^{2}}} \mathrm{~d} x^{1} \cdots \mathrm{d} x^{d} .
\end{aligned}
$$


Since the term $\mathrm{e}^{-\frac{\left(t^{1}\right)^{2}}{2 \sigma^{2}} \cdots-\frac{\left(t^{d}\right)^{2}}{2 \sigma^{2}}}$ is independent of the variables $x^{1}, \cdots, x^{d}$ one can write (23) as

$$
\begin{aligned}
& { }^{*} \mathcal{F}\left(t_{1}, \cdots, t_{d}\right) \\
& =\frac{\sigma^{q}}{(2 \pi)^{q / 2}} \mathrm{e}^{-\frac{\left(t^{1}\right)^{2}}{2 \sigma^{2}} \cdots-\frac{\left(t^{d}\right)^{2}}{2 \sigma^{2}}} \int_{-\infty}^{\infty} \cdots \int_{-\infty}^{\infty} \mathrm{e}^{-\frac{1}{2}\left(\sigma x^{1}-\frac{i t^{1}}{\sigma}\right)^{2}} \cdots \mathrm{e}^{-\frac{1}{2}\left(\sigma x^{d}-\frac{i t^{d}}{\sigma}\right)^{2}} \mathrm{~d} x^{1} \cdots \mathrm{d} x^{d} .
\end{aligned}
$$

One can again make the change of variable $u_{i}=\left(\sigma x^{i}-\frac{i t^{i}}{\sigma}\right)$, so that $\mathrm{d} u_{i}=\sigma \mathrm{d} x^{i}$ and hence (24) becomes

$$
{ }^{*} \mathcal{F}\left(t_{1}, \cdots, t_{d}\right)=\frac{\sigma^{q}}{(2 \pi)^{q / 2} \sigma^{d}} \mathrm{e}^{-\frac{\left(t^{1}\right)^{2}}{2 \sigma^{2}} \cdots-\frac{\left(t^{d}\right)^{2}}{2 \sigma^{2}}} \int_{-\infty}^{\infty} \cdots \int_{-\infty}^{\infty} \mathrm{e}^{-\frac{1}{2} u_{1}^{2}} \cdots \mathrm{e}^{-\frac{1}{2} u_{d}^{2}} \mathrm{~d} u_{1} \cdots \mathrm{d} u_{d} .
$$

Performing the integrals in (25) yield

$$
{ }^{*} \mathcal{F}\left(t_{1}, \cdots, t_{d}\right)=\frac{\sigma^{q}(2 \pi)^{d / 2}}{(2 \pi)^{q / 2} \sigma^{d}} \mathrm{e}^{-\frac{\left(t^{1}\right)^{2}}{2 \sigma^{2}} \cdots-\frac{\left(t^{d}\right)^{2}}{2 \sigma^{2}}} .
$$

Thus, if $d=2 q$ then one finds

$$
{ }^{*} \mathcal{F}\left(t_{1}, \cdots, t_{d}\right)=\frac{(2 \pi)^{q / 2}}{\sigma^{q}} \mathrm{e}^{-\frac{\left(t^{1}\right)^{2}}{2 \sigma^{2}} \cdots-\frac{\left(t^{d}\right)^{2}}{2 \sigma^{2}}} .
$$

In particular, by setting $q=2$ one obtains $d=4$ and thus (16) leads to the Gaussian distribution

$$
\mathcal{G}=\frac{\sigma^{2}}{2 \pi} \mathrm{e}^{-\frac{\sigma^{2}}{2}\left[\left(x^{1}\right)^{2}+\left(x^{2}\right)^{2}+\left(x^{3}\right)^{2}+\left(x^{4}\right)^{2}\right]}
$$

and therefore according to (27) the Fourier transform of $\mathcal{G}$ is given by

$$
\mathcal{G}^{*}=\frac{2 \pi}{\sigma^{2}} \mathrm{e}^{-\frac{1}{2 \sigma^{2}}\left[\left(t^{1}\right)^{2}+\left(t^{2}\right)^{2}+\left(t^{3}\right)^{2}+\left(t^{4}\right)^{2}\right]},
$$

in agreement with (17).

Note the surprising duality relation between $\mathcal{G}$ and $\mathcal{G}^{*}$, namely

$$
\sigma^{2} \leftrightarrow \frac{1}{\sigma^{2}}
$$

which only is fully achieved in $d=4$. In this sense one can say that the 4-dimensions associated with $\mathcal{G}^{*}$ and and 4-dimensions associated with $\mathcal{G}$ are dual to each other. Observe that $\mathcal{G}^{* *} \sim \mathcal{G}$ which is the usual requirement for a duality symmetry. From (14) one knows that $\sigma^{2}=\frac{1}{l_{P} l_{0}}$ so that (30) can be written as

$$
l_{P} l_{0} \leftrightarrow \frac{1}{l_{P} l_{0}}
$$


Thinking about the magnetic monopole $g$ and the electric charge $e$ duality, namely

$$
e \leftrightarrow \frac{n \hbar}{e},
$$

with $g e=n \hbar$, one is tempted to assume that (31) implies a duality of the form

$$
l_{P} l_{0}=n \alpha,
$$

where $\alpha$ is a constant to be determined. Considering that at the present, the Planck length is of the order $l_{P} \sim 10^{-33} \mathrm{~cm}$ and assuming the de Sitter length is of the order of the radius of the Universe $l_{0} \sim 10^{+28} \mathrm{~cm}$, for $n=1$, one obtains that $\alpha$ is of the order of $10^{-5} \mathrm{~cm}^{2}$ which is too large to be identified with any fundamental atomic radius. However, an interesting and attractive possibility is to assume the condition $\alpha \sim 1 \mathrm{~cm}^{2}$, which implies that $l_{0} \sim 10^{+33} \mathrm{~cm}$.

It is remarkable that there exist a relation between the de Sitter length $l_{0}$ and the cosmological constant $\Lambda$, namely (see [8] and references therein)

$$
\frac{2 \Lambda}{(d-1)(d-2)}=\frac{1}{l_{0}^{2}} \text {. }
$$

In $d=4$ one gets

$$
\frac{\Lambda}{3}=\frac{1}{l_{0}^{2}}
$$

Thus, the duality relation (31) can be written as

$$
\left(\frac{\Lambda_{P} \Lambda}{9}\right)^{1 / 2} \leftrightarrow \frac{1}{\left(\frac{\Lambda_{P} \Lambda}{9}\right)^{1 / 2}},
$$

where one has defined

$$
\frac{\Lambda_{P}}{3}=\frac{1}{l_{p}^{2}}
$$

Since one is assuming the formula (33) one sees that $\Lambda_{P} \Lambda=\beta^{2}$, with $\beta^{2}=\frac{9}{n^{2} \alpha^{2}}$ and hence one has discovered a cosmological constant duality

$$
\Lambda \leftrightarrow \frac{1}{\Lambda_{P}}
$$

So, considering the case $n=1$ and $\alpha=1$, since $l_{p}$ is of the order of $10^{-33}$ $\mathrm{cm}$, one finds that $\Lambda_{P}$ is of the order of $10^{66} \mathrm{~cm}^{-2}$ which is, of course, very large, but according to (38) the cosmological constant $\Lambda$ will be very small of the order $10^{-66} \mathrm{~cm}^{-2}$. It is worth mentioning that the type of duality (38) has been previously described in the context of $S$-duality for linearized gravity [9].

\section{Final Remarks}

In the above discussion, we have focused in the de Sitter space with $\Lambda>0$, however similar conclusions must be achieved in the case of anti de Sitter space 
with $\Lambda<0$ (see Refs [4] [5] and references therein). Further, in the usual quantum mechanics, the Fourier transform relate in one to one correspondence the configuration space of $q$-coordinate with the conjugate momentum $p$-coordinates. If there are $n$ such $q$-coordinates there must $n p$-coordinates and this mean that the total phase space must be $(n+n)$-dimensional. Following this route of though one can say that the Fourier transform between the $4 x^{\mu}$ -coordinates and the $4 t^{\alpha}$-coordinates in our approach must lead inevitably to a $(4+4)$-dimensional space-time. In fact, combining (23), (28) and (29) one may write

$$
f^{*}=\hat{f}\left(\mathrm{e}^{-\frac{\sigma^{2} x^{\mu} x^{v} \delta_{\mu v}}{2}+\frac{t^{\mu} t^{\nu} \delta_{\mu v}}{2 \sigma^{2}}}\right),
$$

with $\mu, v=1,2,3,4$. Here,

$$
\begin{aligned}
& \hat{f}\left(\mathrm{e}^{-\frac{\sigma^{2} x^{\mu} x^{v} \delta_{\mu v}}{2}+\frac{t^{\mu} t^{v} \delta_{\mu v}}{2 \sigma^{2}}}\right) \\
& =\frac{\sigma^{2}}{2 \pi} \mathrm{e}^{\frac{t^{\mu} t^{v} \delta_{\mu v}}{2 \sigma^{2}}} \int_{-\infty}^{\infty} \int_{-\infty}^{\infty} \int_{-\infty}^{\infty} \int_{-\infty}^{\infty} \mathrm{e}^{-\frac{1}{2} i x^{\mu} t^{v} \delta_{\mu v}} \mathrm{e}^{-\frac{\sigma^{2} x^{\mu} x^{v} \delta_{\mu v}}{2}} \mathrm{~d} x^{1} \mathrm{~d} x^{2} \mathrm{~d} x^{3} \mathrm{~d} x^{4}
\end{aligned}
$$

and $f^{*}=\frac{2 \pi}{\sigma^{2}}$. So, from (39) one may conclude that the space-time involved may be considered to correspond to a $(4+4)$-dimensional. Therefore, a surprising scenario emerges from our formalism: The point is that our result refers to both vacuum de Sitter space associated with the "macroscopic" $x^{\mu}$-world of 4-dimensional space and the zero-point "energy" linked to the lowest possible energy at that quantum "microscopic" $t^{\alpha}$-world of 4-dimensions, and vice versa. The key result is that these two worlds are related by duality symmetry; a standard deviation $\sigma$ of a $x^{\mu}$-world is dual to the $\frac{1}{\sigma}$ in the $t^{\alpha}$-world. This means that a $x^{\mu}$-world with thin and tall (small $\sigma$ ) Gaussian distribution is dual to wide and low (large $\sigma$ ) Gaussian distribution in the other $t^{\alpha}$-world and vice versa. Moreover, this is verified by observing that the large cosmological constant $\Lambda_{P}$ in the quantum world is dual to the small cosmological constant $\Lambda$ in the classical cosmological world. This seems to explain, in a cosmological context, the smallness of the cosmological constant.

Thinking in the possible geometrization of the duality symmetry in $(4+$ 4)-dimensions space, one is tempted to propose the modified line element

$$
\mathrm{d} s^{2}=-\frac{c^{2} \mathrm{~d} t^{\mu} \mathrm{d} t^{\nu} \delta_{\mu v}}{\sigma^{2} l_{P}^{4}}+\sigma^{2} \mathrm{~d} x^{\mu} \mathrm{d} x^{v} \delta_{\mu \nu} .
$$

Here, we have introduced proper units in order to have $\mathrm{ds}^{2}$ dimensionless, the $t$-coordinates in seconds and $x$-coordinates in centimeters. So, (41) considers explicitly the standard deviation $\sigma$ in a dual form. It remains to explore the full consequences of (41). 
The idea that duality emerges as a key concept in vacuum cosmology in 4-dimensions has been explored before in [10]. Here, we have shown that duality also seems to play a fundamental role in vacuum cosmology in $(4+4)$-dimensions, at both macroscopic and microscopic level. So, one wonders whether duality can be the central mathematical notion in quantum gravity. This route of though shall bring us sooner or later to look for a mathematical structure as a frame for the duality concept. In turns out that such a formalism already exist, namely oriented matroid theory (see Refs. [11]-[17] and references therein). This mathematical framework is a generalization of both graphs and matrices and establishes that every oriented matroid has a dual. This means that although every graph corresponds to a matroid, there are matroids which are not graphic. So, this clarify why the complete graphs $K_{5}$ and $K_{3,3}$ do not have a dual; they have an associated matroid $M\left(K_{5}\right)$ and $M\left(K_{3,3}\right)$ but the corresponding dual matroids $M^{*}\left(K_{5}\right)$ and $M^{*}\left(K_{3,3}\right)$ are not graphic. Thus, oriented matroid theory has entered its key concept: duality. So, one may be interesting for further work to establish the relation between the duality described in this work and oriented matroid theory.

Finally, let us just mention another source of interest for further work. We refer to the surreal number theory. This is a mathematical structure that was discovered by Conway [18] (see also Ref. [19]) and Gonshor [20]. Among its interesting properties of such numbers is that natural, integers and real numbers are contained in the surreal number framework. In Refs. [21] and [22] it was established the main reasons why we believe that such a numbers may be physically interesting. In particular one may be interested in considering surreal number theory in the context of quantum gravity in $(4+4)$-dimensions. Let us clarify this comment. Classical general relativity in $(4+4)$-dimensions can be formulated in $R^{8}$, that is in a continuum 8-dimensional space-time background. So quantizing gravity may be though as the framework, which must imply at mechanism for starting with a continuum differential geometry in 8-dimensions and then discretize such a geometry. But what if we go backwards? That is, we start with a discrete mathematical structure of surreal numbers $\mathcal{S}$ and we end up with a continuum 8-dimensional manifold based on $R$. Consider the set [18]

$$
s=\left\{S_{L} \mid S_{R}\right\}
$$

and call $S_{L}$ and $S_{R}$ the left and right sets of $s$, respectively. Surreal numbers are defined in terms of two axioms:

Axiom 1. Every surreal number corresponds to two sets $S_{L}$ and $S_{R}$ of previously created numbers, such that no member of the left set $S_{L} \in S_{L}$ is greater or equal to any member $S_{R}$ of the right set $S_{R}$.

Let us denote by the symbol $\nsucceq$ the notion of no greater or equal to. So the axiom establishes that if $s$ is a surreal number then for each $s_{L} \in S_{L}$ and $s_{R} \in S_{R}$ one has $s_{L} \nsucceq s_{R}$. This is denoted by $S_{L} \nsucceq S_{R}$.

Axiom 2. One number $s=\left\{S_{L} \mid S_{R}\right\}$ is less than or equal to another number $s^{\prime}=\left\{S_{L}^{\prime} \mid S_{R}^{\prime}\right\}$ if and only if the two conditions $S_{L} \nsucceq s^{\prime}$ and $s \nsupseteq S_{R}^{\prime}$ are satis- 
fied.

This can be simplified by saying that $s \leq s^{\prime}$ if and only if $S_{L} ¥ s^{\prime}$ and $s \nsupseteq S_{R}^{\prime}$.

Observe that Conway definition relies in an inductive method; before a surreal number $x$ is introduced one needs to know the two sets $S_{L}$ and $S_{R}$ of surreal numbers. Following Conway algorithm one finds numbers, of the type

$$
s=\frac{m}{2^{n}}
$$

where $m$ is an integer and $n$ is a natural number, $n>0$. Of course, the numbers (43) are dyadic rationals which are dense in the real $R$.

A different but equivalent definition of surreal numbers is due to Gonshor [20]:

Definition 1. A surreal number is a function $f$ from initial segment of the ordinals into the set $\{+,-\}$.

For instance, if $f$ is the function so that $f(1)=+, f(2)=+, f(3)=-$, $f(4)=+$ then $f$ is the surreal number $(++-+)$.

It can be shown that the positive sectors of surreal numbers are given by [23]

$$
\mathcal{J}_{(+)}\left(l_{1}, l_{2}\right)= \begin{cases}(\mathrm{I}) l_{1}, & \text { if } l_{2}-l_{1}=0, \\ (\mathrm{II}) l_{1}-\frac{1}{2}, & \text { if } l_{2}-l_{1}=1 \\ (\text { III }) l_{1}-\frac{1}{2} \pm \sum_{k=1}^{l_{2}-\left(l_{1}+1\right)} \frac{1}{2^{k+1}}, & \text { if } l_{2}-l_{1}>1\end{cases}
$$

Here, the numbers $l_{1}$ and $l_{2}$ take values in the set $\{1,2,3, \ldots\}$. The negative sector is obtained as $\mathcal{J}_{(-)}\left(l_{1}, l_{2}\right)=-\mathcal{J}_{(+)}\left(l_{1}, l_{2}\right)$. This implies that from (I) and (II) one gets $\mathcal{J}_{(+)}(1,1)=1, \mathcal{J}_{(+)}(1,2)=\frac{1}{2}$ and from (III) one obtains $\mathcal{J}_{(+)}(1,3)=\left\{\frac{1}{4}, \frac{3}{4}\right\}, \mathcal{J}_{(+)}(1,4)=\left\{\frac{1}{8}, \frac{3}{8}, \frac{5}{8}, \frac{7}{8}\right\}$ and so on. Since $\mathcal{J}_{(-)}\left(l_{1}, l_{2}\right)=-\mathcal{J}_{(+)}\left(l_{1}, l_{2}\right)$ one also has $\mathcal{J}_{(-)}(1,1)=-1, \quad \mathcal{J}_{(=)}(1,2)=-\frac{1}{2}$ and $\mathcal{J}_{(=)}(1,3)=\left\{-\frac{1}{4},-\frac{3}{4}\right\}, \mathcal{J}_{(+)}(1,4)=\left\{-\frac{1}{8},-\frac{3}{8},-\frac{5}{8},-\frac{7}{8}\right\}$ and so on.

How can be related the present formalism to surreal numbers? The first thing that we would like to highlight is that just as in $(4+4)$-world the duality symmetry is a key concept, the starting point in both the Conway and the Gonshor formulation is duality scenario. In the case of Conway formulation we see from (42) that a surreal numbers is defined in terms of dual sets $S_{L}$ and $S_{R}$, left and right sets of $s$, respectively. While in the Gonshor definition of surreal number is a function $f$ from initial segment of the ordinals into the dual set $\{+,-\}$. Since these two formulations are equivalents one conclude the key concept in both is duality. The second observation is that the zero-point "energy" in (10), namely $\varepsilon=\frac{l_{P} \omega}{2}$ remind us the spin structure of $\frac{1}{2}$-spin in the sense that 
$s=\frac{\hbar}{2}$. However, theoretically there are many type of fermions, including $s=\frac{3 \hbar}{2}$ (gravitino) and $s=\frac{5 \hbar}{2}$. And these spin structures start looking as dyadic rational (43) arising in the surreal number algorithm. In fact, this kind of observation motivate to propose the dyadic rational $\frac{m}{2^{n}}$-spin as an alternative tool for quantum gravity and dark matter [21] [22] and [24]. Thus, it is tempted to propose for each coordinate in $(x, y, z, \xi)=\left(x^{1}, x^{2}, x^{3}, x^{4}\right)$ the equation

$$
\frac{\mathcal{J}_{(+)}\left(l_{1}, l_{2}\right) l_{P} \omega}{2}=-\frac{l_{P}^{2}}{2} \frac{1}{\varphi_{x}} \frac{\mathrm{d}^{2} \varphi_{x}}{\mathrm{~d} x^{2}}+\frac{\mathcal{J}_{(+)}^{2}\left(l_{1}, l_{2}\right) \omega^{2} x^{2}}{2},
$$

which generalize the zero-point "energy" for the harmonic oscillator (12). This equation can be rewritten as

$$
\frac{l_{P} \tilde{\omega}}{2}=-\frac{l_{P}^{2}}{2} \frac{1}{\varphi_{x}} \frac{\mathrm{d}^{2} \varphi_{x}}{\mathrm{~d} x^{2}}+\frac{\tilde{\omega}^{2} x^{2}}{2},
$$

with $\tilde{\omega}=\mathcal{J}_{(+)}\left(l_{1}, l_{2}\right) \omega=\frac{\mathcal{J}_{(+)}\left(l_{1}, l_{2}\right)}{l_{0}}$. Hence the general solution of (45) must be similar to (28), namely

$$
\mathcal{G}=\frac{\tilde{\sigma}^{2}}{2 \pi} \mathrm{e}^{-\frac{\tilde{\sigma}^{2} \mathcal{J}_{(+)}\left(l_{1}, l_{2}\right)}{2}\left[\left(x^{1}\right)^{2}+\left(x^{2}\right)^{2}+\left(x^{3}\right)^{2}+\left(x^{4}\right)^{2}\right]},
$$

with $\tilde{\sigma}^{2}=\frac{\tilde{\omega}}{l_{P}}$. This means that the Fourier transform of $\mathcal{G}$ must be given by

$$
\mathcal{G}^{*}=\frac{2 \pi}{\tilde{\sigma}^{2}} \mathrm{e}^{-\frac{1}{2 \tilde{\sigma}^{2}}\left[\left(t^{1}\right)^{2}+\left(t^{2}\right)^{2}+\left(t^{3}\right)^{2}+\left(t^{4}\right)^{2}\right]} .
$$

Observe that in this case the zero-point "energy" will be

$$
\tilde{\varepsilon}\left(l_{1}, l_{2}\right)=l_{P} \tilde{\omega}=l_{P} \omega \mathcal{J}_{(+)}\left(l_{1}, l_{2}\right),
$$

with $\mathcal{J}_{(+)}\left(l_{1}, l_{2}\right)$ given by (44). One observes that considering the point (II) in (44) the expression (49) exactly reproduces the usual eigenvalues of the energy of the harmonic oscillator, with $n=l_{1}-1$. We think that this rough derivation of (49) may motivate the subject for further work.

Let us conclude this work by summarizing our main results. First, by taking a duality transformation as a key symmetry we explore some aspects a cosmological model of four time and four space dimensions $((4+4)$-dimensions). In particular, we show that, at the level of zero-point energy in a de Sitter space, a Fourier transform of a Gaussian distribution in four dimensions leads to a dual Gaussian distribution also in four dimensions, with duality transformation $\sigma \rightarrow \frac{1}{\sigma}$, in the standard deviation $\sigma$. Moreover, we show that as a consequence of such a duality in $\sigma$ a duality of the cosmological constant $\Lambda$, associated with the Sitter space, can be obtained. Finally, since duality symmetry is a fundamental concept in both the oriented matroid theory as well as the surreal 
number theory we roughly explain how these mathematical theories can be connected to the present work.

\section{Acknowledgements}

JA Nieto would like to thank the Mathematical, Computational \& Modeling Sciences Center of the Arizona State University where part of this work was developed. We would like to thank to Clarissa Avila for helpful comments. This work was partially supported by PROFAPI 2013.

\section{Conflicts of Interest}

The authors declare no conflicts of interest regarding the publication of this paper.

\section{References}

[1] Green, M.B., Schwarz, J.H. and Witten, E. (1987) Superstring Theory I and II. Cambridge University Press, Cambridge.

[2] Duff, M.J. (1996) International Journal of Modern Physics A, 11, 5623-5641. https://doi.org/10.1142/S0217751X96002583

[3] Hull, C.M. (1998) JHEP, 11, 17. https://doi.org/10.1088/1126-6708/1998/11/017

[4] Yan, M.-L. (2015) De Sitter Invariant Special Relativity. University of Science and Technology of China, Hefei.

[5] Licata, I., Chiatti, L. and Benedetto, E. (2017) De Sitter Projective Relativity. Springer Briefs in Physics, Springer, Berlin. https://doi.org/10.1007/978-3-319-52271-5

[6] Nieto, J.A. and Espinoza, M. (2017) International Journal of Geometric Methods in Modern Physics, 14, Article ID: 1750014. https://doi.org/10.1142/S0219887817500141

[7] Nieto, J.A. and Madriz, E. (2019) Physica Scripta, 94, Article ID: 115303. https://doi.org/10.1088/1402-4896/ab2d96

[8] Avila, G., Castillo, S.J. and Nieto, J.A. (2016) Journal of Interdisciplinary Mathematics, 19, 955-975.

[9] Nieto, J.A. (1999) Physics Letters A, 262, 274-281. https://doi.org/10.1016/S0375-9601(99)00702-1

[10] Tkach, V.I., Socorro, J., Rosales, J.J. and Nieto, J.A. (1999) Physical Review D, 60, Article ID: 067503. https://doi.org/10.1103/PhysRevD.60.067503

[11] Nieto, J.A. (2004) Advances in Theoretical and Mathematical Physics, 8, 177-188. https://doi.org/10.4310/ATMP.2004.v8.n1.a4

[12] Nieto, J.A. (2006) Advances in Theoretical and Mathematical Physics, 10, 747-757. https://doi.org/10.4310/ATMP.2006.v10.n5.a5

[13] Nieto, J.A. (2004) Journal of Mathematical Physics, 45, 285. https://doi.org/10.1063/1.1625416

[14] Nieto, J.A. (2014) Nuclear Physics B, 883, 350-372. https://doi.org/10.1016/j.nuclphysb.2014.04.001

[15] Nieto, J.A. and Marn, M.C. (2000) Journal of Mathematical Physics, 41, 7997. https://doi.org/10.1063/1.1319518

[16] Nieto, J.A. (2013) Physics Letters B, 718, 1543-1547. 
https://doi.org/10.1016/j.physletb.2012.12.034

[17] Nieto, J.A. (2010) Physics Letters B, 692, 43-46. https://doi.org/10.1016/j.physletb.2010.07.010

[18] Conway, J.H. (1976) On Number and Games. London Mathematical Society Monographs. Academic Press, Cambridge.

[19] Knuth, D.E. (1974) Surreal Numbers: How Two Ex-Students Turned on to Pure Mathematics and Found Total Happiness: A Mathematical Novelette. AddisonWesley Publishing Co., Boston.

[20] Gonshor, H. (1986) An Introduction to the Theory of Surreal Numbers. London Mathematical Society Lectures Notes Series, Vol. 110, Cambridge Univ. Press, Cambridge. https://doi.org/10.1017/CBO9780511629143

[21] Nieto, J.A. (2016) Journal of Modern Physics, 7, 2164-2176. https://doi.org/10.4236/jmp.2016.715188

[22] Nieto, J.A. (2018) Frontiers in Physics, 6, Article No. 106. https://doi.org/10.3389/fphy.2018.00106

[23] Avalos-Ramos, C., Felix-Algandar, J.A. and Nieto, J.A. (2020) IOSR Journal of Mathematics, 16, 35 .

[24] Aviles-Niebla, C., Nieto-Marn, P.A. and Nieto, J.A. (2020) International Journal of Geometric Methods in Modern Physics, 17, Article ID: 2050180.

https://doi.org/10.1142/S0219887820501807 